\title{
Review of modeling and control strategy of thermostatically controlled loads for virtual energy storage system
}

\author{
Kang Xie, Hongxun Hui and Yi Ding*
}

\begin{abstract}
The increasing penetration of renewable energy sources (RESs) brings more power generation fluctuations into power systems, which puts forward higher requirement on the regulation capacities for maintaining the power balance between supply and demand. In addition to traditional generators for providing regulation capacities, the progressed information and communication technologies enable an alternative method by controlling flexible loads, especially thermostatically controlled loads (TCLs) for regulation services. This paper investigates the modeling and control strategies of aggregated TCLs as the virtual energy storage system (VESS) for demand response. First, TCLs are modeled as VESSs and compared with the traditional energy storage system (ESS) to analyze their characteristic differences. Then, the control strategies of VESS are investigated in microgrid and main grid aspects, respectively. It shows that VESS control strategies can play important roles in frequency regulation and voltage regulation for power systems' stability. Finally, future research directions of VESS are prospected, including the schedulable potential evaluation, modeling of TCLs, hierarchical control strategies of VESS considering ESSs and RESs and reliability and fast response in frequency control for VESS.
\end{abstract}

Keywords: Thermostatically controlled loads (TCLs), Virtual energy storage system (VESS), Modeling method, Control strategy

\section{Introduction}

The rapidly growing economy nowadays is consuming more fossil energies, causing a worldwide environmental pollution [1]. Therefore, renewable energy sources (RESs) are paid more attention to and deployed rapidly around the world $[2,3]$. For example, the cumulative installed capacities of wind power and photovoltaic (PV) in China have increased to $184 \mathrm{GW}$ and $174 \mathrm{GW}$ by 2018 , respectively $[4,5]$. However, the power generation by wind power and PV is affected significantly by realtime wind and weather, which have lots of uncertainties and bring more intermittence and fluctuations to power systems [6]. As a result, more traditional power plants (e.g., thermal power plants) need to be built to provide more regulation power for reducing fluctuation risks brought by RESs, and maintaining the power balance between supply and demand.

\footnotetext{
* Correspondence: yiding@zju.edu.cn

Electrical Engineering College of Zhejiang University, Hangzhou 310027, China
}

Thermostatically controlled loads (TCLs), such as air conditionings (ACs), are consuming more electricity [7]. For example, according to the statistics [8], ACs have accounted for around $30 \sim 40 \%$ of peak loads during summer in China, even 50\% in Shanghai and Beijing. Besides, the progressed information and communication technologies nowadays have provided better infrastructure to provide regulation services for power systems by adjusting the power consumption of TCLs remotely or autonomously [9], named as demand response (DR) [10]. It has been proved in many previous studies that DR plays a significant role in reducing power systems' fluctuation risks caused by uncertainties of RESs' output [11]. Therefore, to some extent, DR can replace the construction of new power plants to meet higher requirement of regulation capacities. With the phasing out of traditional power plants, the DR will become more important [12].

Previously, many studies have been done on the modeling and control of TCLs. In terms of modeling, Qi et al. 
model the aggregated TCLs consisting of electric water heaters based on the thermodynamic process to provide frequency regulation for power systems [13]. The electricity consumption model of aggregated ACs is proposed in [14] by Monte Carlo method based on the principle of heat balance. This model can help ACs provide reserve capacity for the power system. Reference [15] studies the virtual energy storage system (VESS) model of TCLs based on the heat storage capacity of buildings to participate in DR. Besides, many papers also propose the TCL model based on the principle of building thermal balance [16-18].

Moreover, based on the above modeling methods, the control strategies of TCLs are also studied. For example, the two-layer flexible optimal strategy is proposed by Wang et al., who minimize the users' electricity charges and maximize the overall coefficient of performance (COP) of chillers, considering the ambient temperature, the electricity price information and the users' comfort [19]. In [20], the hybrid control strategy consisting of dead band control and hysteresis control is presented to provide frequency regulation service for the power system. Moreover, reference [21] establishes the multiobjective optimal scheduling model considering ACs and batteries to reduce the peak-valley difference of loads and the operating cost of the distribution network. In [22], a dynamic economic dispatch strategy is proposed by equating TCL to the VESS to reduce operation cost and ensure users' comfort at the same time.

However, even though many studies have been done on the modeling and control methods of TCLs, the equivalence methods of TCL to VESS is insufficient. It is just this paper attempt to investigate and fill.

As the overall structure of this paper in Fig. 1, the remaining of this paper is organized as follows. Section 2 analyses the equivalent models of the TCL to the VESS, including the individual TCL and aggregated TCLs. Section 3 focuses on the control strategies of VESS. Suggestions on VESS are put forward for future research in Section 4. Section 5 concludes this paper.

\section{Modeling of virtual energy storage system \\ 2.1 Modeling of energy storage system}

Energy storage systems (ESSs) can store electricity energy and deliver it to loads when needed. It means that the ESS can transfer energy from one time to another, or from one place to another. Therefore, the ESS is regarded as an important device to shift peak loads, improve the power quality and enhance the power system reliability $[23,24]$.

The energy transfer process of the ESS meets energy conservation formula [25], and it can be expressed as

$$
Q_{t}^{j}=Q_{o}^{j}+\eta_{c h}^{j} \cdot \sum_{i=1}^{t} Q_{c h, i}^{j}-\sum_{i=1}^{t} Q_{d i s, i}^{j}
$$

where $Q_{t}^{j}$ and $Q_{o}^{j}$ are the stored energy in the $j$-th ESS at time $t$ and the initial time, respectively. $Q_{c h, i}^{j}$ and $Q_{d i s, i}^{j}$ are the charging energy and discharging energy of the $j$ th ESS for the $i$-th time, respectively. $\eta_{c h}^{j}$ is the charging efficiency of the $j$-th ESS. Therefore, based on the Eq. (1), the energy increment of the $j$-th ESS depends on the difference between the charging energy and the discharging energy of the ESS.

Moreover, in order to ensure the safety operation, the ESS is subject to some constraints [26], including the power constraints and the state of charge (SOC) constraints, which can be expressed as

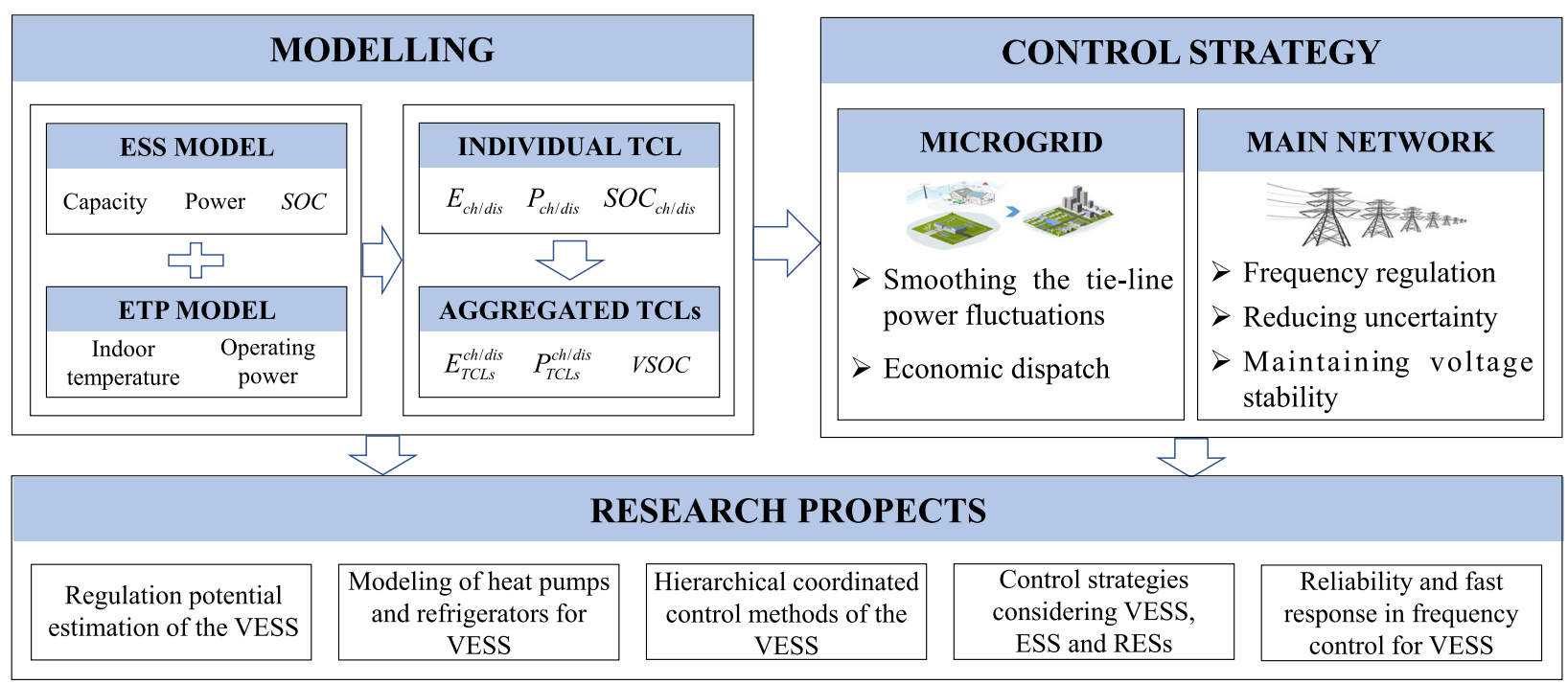

Fig. 1 Overall structure of this paper 


$$
\begin{aligned}
& \underline{P_{c h}^{j}} \leq P_{t, c h}^{j} \leq \overline{P_{c h}^{j}} \\
& \underline{P_{d i s}^{j}} \leq P_{t, d i s}^{j} \leq \overline{P_{d i s}^{j}} \\
& \underline{S O C^{j}} \leq S O C_{t}^{j} \leq \overline{S O C^{j}}
\end{aligned}
$$

where $P_{t, c h}^{j}$ and $P_{t, d i s}^{j}$ are the charging and discharging power of the $j$-th ESS at time $t . \underline{P}_{c h}^{j}$ and $\underline{P_{d i s}^{j}}$ are the minimum values of the charging and discharging power of the $j$-th ESS. $\overline{P_{c h}^{j}}$ and $\overline{P_{d i s}^{j}}$ are the maximum values of the charging and discharging power of the $j$-th ESS. $\underline{S O C^{j}}$ and $\overline{S O C^{j}}$ are the minimum and maximum values of the $S O C$ for the $j$-th ESS. SOC $C_{t}^{j}$ is the state of charge of the $j$-th ESS at time $t$, which represents the percentage of remaining electricity of the ESS. It can be expressed as [27].

$$
S O C_{t}^{j}=\frac{Q_{t}^{j}}{Q_{n}^{j}}
$$

where $Q_{n}^{j}$ is the rated capacity of the $j$-th ESS.

\subsection{ETP model of the TCL}

The equivalent thermal parameter (ETP) model [28-31] has been widely used in the modeling of the thermostatically controlled load (TCL), which depicts the transfer and dissipation of heat energy in a room. The first order ETP model can be expressed by an equivalent circuit, as shown in Fig. 2. It equates the heat transfer to the electricity transfer [31]. Therefore, these physical quantities can be calculated by ohm's law. $Q_{T C L}$ is the refrigerating or heating capacity of the TCL. $T_{i n}$ and $T_{o}$ are the internal temperature and ambient temperature, respectively. $R_{T C L}$ and $C_{T C L}$ are the equivalent thermal resistance and thermal capacity, respectively.

Based on the circuit principle, the ETP model in Fig. 2 can be described as

$$
\frac{d T_{i n}(t)}{d t}=\frac{Q_{T C L}(t)}{C_{T C L}}-\frac{T_{i n}(t)-T_{o}}{R_{T C L} \cdot C_{T C L}}
$$

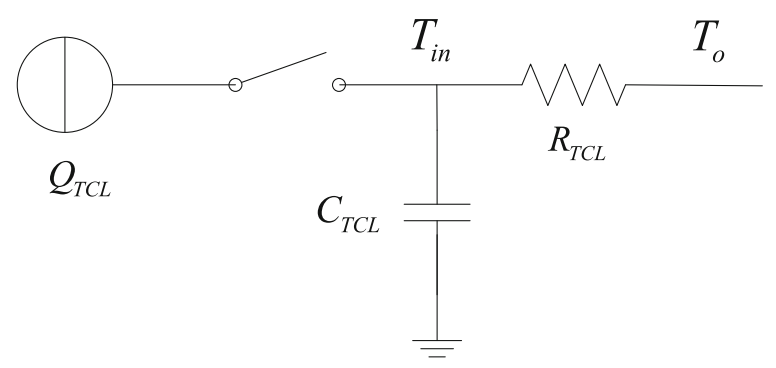

Fig. 2 First order ETP model
On this basis, the varying curves of the indoor temperature and the TCL's power consumption in heating mode can be calculated, as shown in Fig. 3. It is assumed that the initial indoor temperature is equal to the ambient temperature $T_{o}$. When the TCL is turned on, the indoor temperature $T_{i n}$ will go up until it reaches the upper limit $T_{u p}$. Then the TCL will turn off at time $t_{u p}$, and the indoor temperature will begin to drop down. When the indoor temperature decrease to the lower limit $T_{\text {low }}$ at time $t_{\text {low }}$, the TCL will start again to maintain the indoor temperature in the dead band $\left[T_{l o w}, T_{u p}\right]$ [32]. In fact, $T_{\text {up }}$ and $T_{\text {low }}$ are related to the set temperature $T_{\text {set }}$, which can be expressed as

$$
\begin{aligned}
& T_{\text {low }}=T_{\text {set }}-\delta \\
& T_{\text {up }}=T_{\text {set }}+\delta
\end{aligned}
$$

where $\delta$ is the maximum deviation range of the indoor temperature.

As shown in Fig. 3, the TCL's power depends on the indoor temperature, which can be expressed as

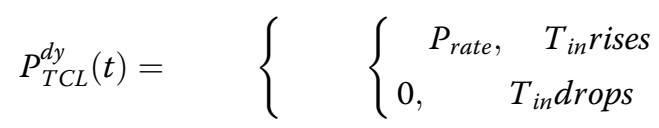

where $P_{\text {rate }}$ is the rated power of the TCL [33].

If the indoor temperature reaches the dead band [ $T_{\text {low }}$, $\left.T_{u p}\right]$, the heat transfer process can be regarded as reaching the steady state [34]. It means that the average heat output of the TCL is equal to the average heat dissipation during steady state. Based on the Eq. (6), the average heat output of the TCL in the steady state can be expressed as

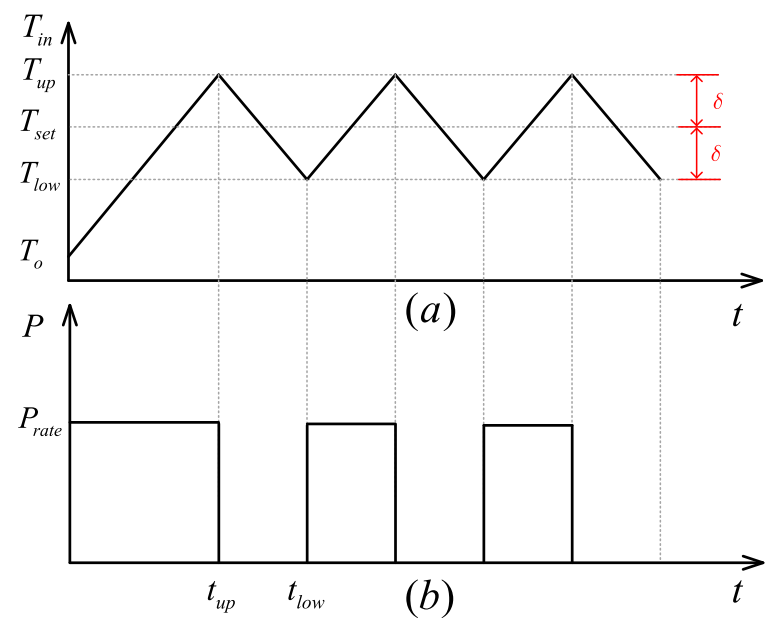

Fig. 3 Operation characteristics of the TCL in heating mode: (a) the varying curves of the indoor temperature and (b) the varying curves of the TCL's power consumption 
Table 1 The typical values of the thermal parameters

\begin{tabular}{llll}
\hline Parameter name & Parameter symbol & Parameter value & Parameter unit \\
\hline Equivalent thermal resistance & $R_{T C L}$ & $1 \sim 3$ & $\mathrm{~W} /\left({ }^{\circ} \mathrm{C} \cdot \mathrm{m}^{2}\right)$ \\
Equivalent thermal capacity & $C_{T C L}$ & $47 \sim 230$ & $\mathrm{~kJ} /\left({ }^{\circ} \mathrm{C} \cdot \mathrm{m}^{2}\right)$ \\
Coefficient of performance & $\eta$ & $2.6 \sim 3.6$ & - \\
\hline
\end{tabular}

$$
Q_{T C L}^{s s}=\frac{T_{s e t}-T_{o}}{R_{T C L}}
$$

Therefore, the average power of the TCL in the steady state can be calculated as

$$
P_{T C L}^{s s}(t)=\frac{Q_{T C L}^{s s}}{\eta}=\frac{T_{\text {set }}-T_{o}}{R_{T C L} \eta}
$$

where $\eta$ is the coefficient of performance $(C O P)$ [8], also known as the energy efficiency ratio (EER).

For ACs, the typical values [35] of the thermal parameters of the TCLs are in Table 1.

\subsection{Equivalence of the individual TCL to VESS}

Based on the ETP model in subsection 2.2, the operating power of the TCL can also be changed to provide regulation power by adjusting the set temperature or switching the operating state from on to off. Due to the thermal characteristics of the room [36], the indoor temperature will not be impacted seriously if the TCL's operating power is adjusted within short period of time. Therefore, the room and the TCL (i.e., the ETP model) can be regarded as an ESS to provide regulation services for the power system, named as virtual energy storage system (VESS) in this paper.

As shown in Figs. 4 and 5, it is assumed that the indoor temperature has been in a steady state before changing the set temperature, i.e., the period $\left[t_{0}, t_{1}\right]$. Therefore, the power consumption of the TCL can be expressed as Eq. (11).

A. Charging process of the VESS.

As shown in Fig. 4, after receiving the control signal from the user or the aggregator at $t_{1}$, the TCL's set temperature is changed from $T_{\text {set } 0}$ to $T_{\text {set } 1}$. Because the indoor temperature $T_{\text {set } 0}$ is below $T_{\text {set } 1}$, the TCL will start running at the rated power to raise the indoor temperature until $T_{s e t 1}$. From the perspective of the power system, the operating power of the TCL raises from $P_{\text {set } 0}^{s s}$ to $P_{\text {rate }}$. This process can be regarded as the charging process of the VESS.

The average operating power of the new steady state at $T_{\text {set } 1}$ is $P_{\text {set } 1}^{s s}$. According to Eq. (11), $P_{\text {set } 1}^{s s}$ is larger than $P_{s e t 0}^{s s}$ since $T_{\text {set } 1}$ is larger than $T_{\text {set }}$. It can be regarded that the charging power is $P_{\text {set } 1}^{s s}-P_{\text {set } 0}^{s s}$ during the new steady state.

When the TCL reaches the maximal participation time in the regulation service at $t_{3}$, the set temperature will

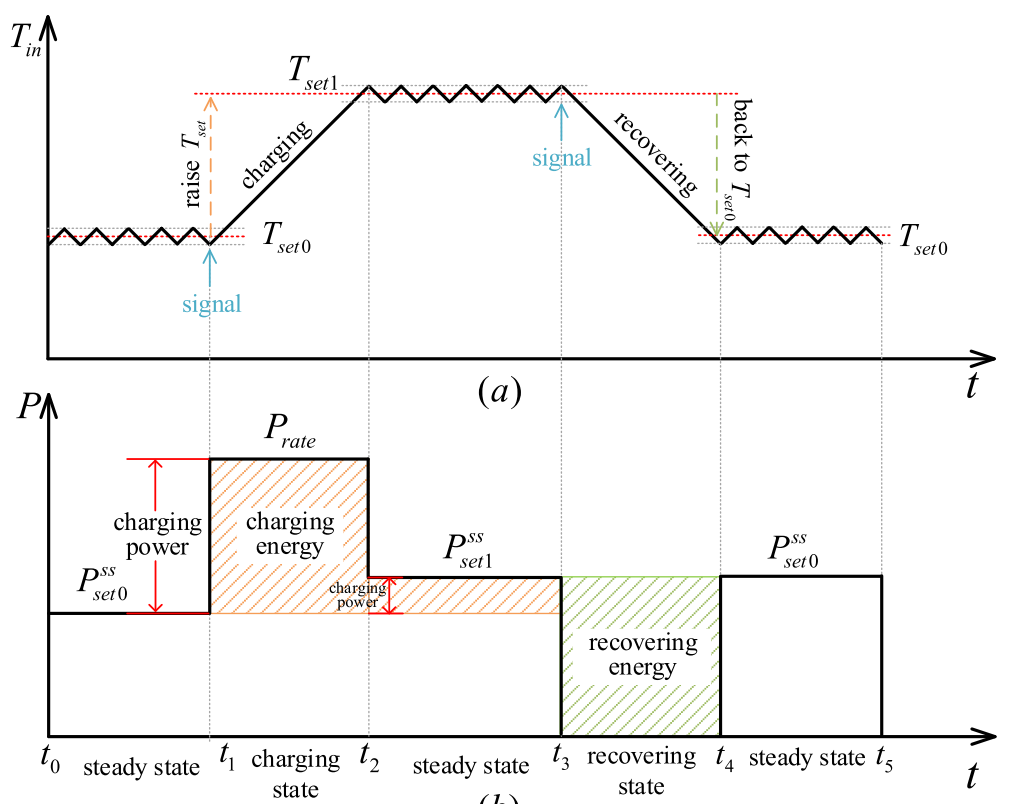

(b)

Fig. 4 Charging process of the VESS in heating mode: (a) the varying curve of the indoor temperature and (b) the varying curve of the VESS' power consumption 


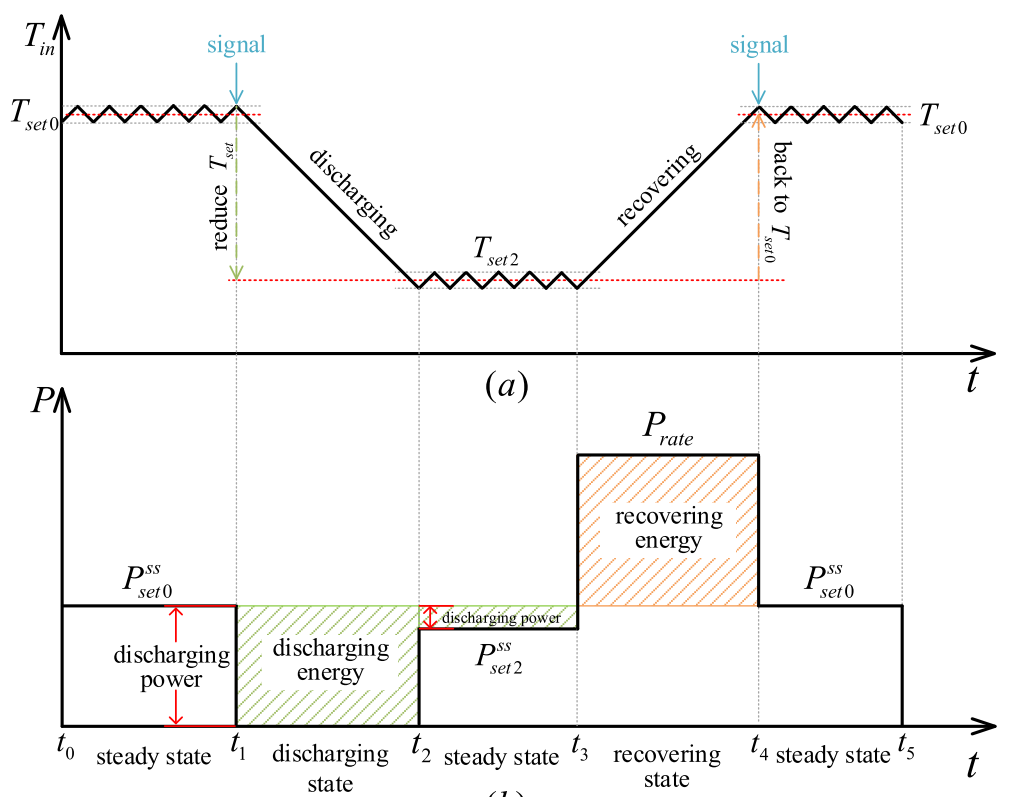

(b)

Fig. 5 Discharging process of the VESS in heating mode: (a) the varying curve of the indoor temperature and (b) the varying curve of the VESS' power consumption

be reset to the original set point $T_{\text {seto }}$ [37]. At that time, the charging process is over, and the TCL enters the recovery process. Finally, the indoor temperature recovers to the original set point $T_{\text {set } 0}$ at $t_{4}$. It can be considered that the charging energy during $t_{1} \sim t_{3}$ dissipates in the period of $t_{3} \sim t_{4}$.

Therefore, the charging power of the VESS has two stages and can be described as

$$
P_{c h}(t)= \begin{cases}P_{\text {rate }}-P_{\text {sst }}^{s s}, & t_{1} \leq t \leq t_{2} \\ P_{\text {set } 1}^{s s}-P_{\text {set } 0}^{s s}, & t_{2} \leq t \leq t_{3}\end{cases}
$$

Based on the Eq. (12), the charging energy of the VESS can be expressed as

$$
E_{c h}(t)=\left\{\begin{array}{rr}
\left(P_{\text {rate }}-P_{\text {set }}^{s s}\right) \cdot\left(t-t_{1}\right), & t_{1} \leq t<t_{2} \\
\left(P_{\text {rate }}-P_{\text {set } 0}^{s s}\right) \cdot\left(t_{2}-t_{1}\right)+\left(P_{\text {set } 1}^{s s}-P_{\text {set } 0}^{s s}\right) \cdot\left(t-t_{2}\right), & t_{2} \leq t<t_{3}
\end{array}\right.
$$

The maximal charging time is from $t_{1}$ to $t_{3}$. Therefore, the maximal charging energy of the VESS can be calculated as

$$
E_{c h}^{\max }=\left(P_{\text {rate }}-P_{s e t 0}^{s s}\right) \cdot\left(t_{2}-t_{1}\right)+\left(P_{s e t 1}^{s s}-P_{s e t 0}^{s s}\right) \cdot\left(t_{3}-t_{2}\right)
$$

Based on the Eq. (13) and Eq. (14), the state of charge $(S O C)$ of the VESS during the charging process can be described as

$$
S O C_{c h}=\frac{E_{c h}}{E_{c h}^{\max }}
$$

During the charging process, some heat will dissipate due to temperature difference between the indoor and outdoor. Therefore, the energy brought by thermostatically controlled loads (TCLs) cannot be completely converted into indoor heat. Assuming the total dissipated energy during the charging process is $E_{c h}^{\text {dissip }}$, the charging efficiency $\eta_{c h}^{V E S S}$ can be expressed as

$$
\eta_{c h}^{V E S S}=\frac{E_{c h}^{\max }-E_{c h}^{d i s s i p}}{E_{c h}^{\max }}
$$

where $E_{c h}^{\text {dissip }}$ is related to the characteristics of the VESS such as the equivalent thermal resistance $R_{T C L}$ and thermal capacity $C_{T C L}$ and temperature difference between the indoor and outdoor. Because the temperature difference between the indoor and outdoor is always changing during charging process, $\eta_{c h}^{V E S S}$ will not be constant.

B. Discharging process of the VESS.

As shown in Fig. 5, similar to the charging process, when the control signal is received at $t_{1}$, the set temperature is reduced from $T_{\text {set } 0}$ to $T_{\text {set } 2 \text {. Because the }}$ indoor temperature $T_{\text {set } 0}$ is higher than $T_{\text {set } 2}$, the TCL is switched off and the operating power is decreased to zero. This process can be regarded that the VESS is discharging to the power system. 
When the indoor temperature reaches $T_{\text {set } 2}$ at $t_{2}$, the TCL enters the new steady state. According to Eq. (11), $P_{\text {set } 2}^{s s}$ is smaller than $P_{\text {set } 0}^{s s}$. It means that the discharging power is $P_{\text {set } 0}^{s s}-P_{\text {set } 2}^{s s}$ during the new steady state. Similar to Fig. 4 , the discharging process is over at $t_{3}$ and the TCL begins to recover to the original state.

Therefore, the discharging power of the VESS can be described as

$$
P_{\text {dis }}(t)=\left\{\begin{array}{lr}
P_{\text {set } 0}^{s s} & t_{1} \leq t \leq t_{2} \\
P_{\text {set } 0}^{s s}-P_{\text {set } 2}^{s s} & t_{2} \leq t \leq t_{3}
\end{array}\right.
$$

On this basis, the discharging energy can be expressed as

$$
E_{\text {dis }}(t)=\left\{\begin{array}{lr}
P_{s e t 0}^{s s} \cdot\left(t-t_{1}\right), & t_{1} \leq t<t_{2} \\
P_{\text {set } 0}^{s s} \cdot\left(t_{2}-t_{1}\right)+\left(P_{\text {set } 0}^{s s}-P_{\text {set } 2}^{s s}\right) \cdot\left(t-t_{2}\right) & t_{2} \leq t \leq t_{3}
\end{array}\right.
$$

The maximal charging energy of the VESS is

$$
E_{\text {dis }}^{\max }=P_{\text {set } 0}^{s s} \cdot\left(t_{2}-t_{1}\right)+\left(P_{\text {set } 0}^{s s}-P_{\text {set } 2}^{s s}\right) \cdot\left(t_{3}-t_{2}\right)
$$

Based on the Eq. (18) and Eq. (19), the SOC of the VESS during the discharging process can be described as

$$
S O C_{d i s}=\frac{E_{d i s}}{E_{d i s}^{\max }}
$$

During the discharging process, the set temperature will be reduced to $T_{\text {set } 2}$ and the operating power will become 0 . The energy stored in the VESS will dissipate to the outside until the indoor temperature reaches to $T_{\text {set } 2}$. In other words, the discharging process is the heat dissipation process. Therefore, the discharging efficiency $\eta_{\text {dis }}^{V E S S}$ is $100 \%$.

In summary, the ETP model of the TCL can be equivalent to VESS. However, the above analysis is based the regular TCLs, whose motors are binary and can be controlled between on- and off-state. For TCLs with inverter and variable frequency functions [38], the operating power can be adjusted flexibly [39]. Therefore, the VESS of these TCLs with variable frequency functions can be modified and controlled more flexibly [40], which needs further study in the future.

\subsection{The comparisons between the ESS, authentic TCL model and the VESS}

\section{A. ESS and VESS.}

Based on the above analysis of the ESS in subsection 2.1 and the VESS in subsection 2.3, the ESS and VESS have lots of similar characteristics. However, there are still some differences between the ESS and VESS.
First, different from the ESS, the charging and discharging power of the VESS depends on the variation of the operating power of TCLs. Secondly, the ESS capacity is decided by its physical or chemical energy storage characteristics, while the VESS' stored energy varies with the change of the set temperature and the participation time. Thirdly, the SOC of the ESS depends on how much energy has been stored [41]. However, the SOC of the VESS depends on the stored energy, the set temperature and the maximal participation time. Besides, the charging efficiency $\eta_{c h}^{V E S S}$ and discharging efficiency $\eta_{d i s}^{V E S S}$ of ESS mainly depend on the physical characteristics itself and there is a big difference for the efficiency of VESS according to Section 2.3. In a word, the characteristic parameters of the VESS are more complex than those of the ESS. The comparisons are shown in detail in Table 2.

B. Authentic TCL model and VESS.

Authentic TCL model describes operating process of TCLs based on the change of indoor temperature, while the VESS model shows it from the perspective of indoor thermal energy. The VESS model has simpler and clearer formulas by comparing Eq. (6)-(11) of authentic TCL model with Eq. (12)-(20) of VESS. Therefore, the complexity of VESS model is lower.

The authentic TCL model is complex, and in order to control with ESS, many equivalence approaches (e.g. continuous state constraints-based method) need to be used. By contrast, the VESS model can explain operating process clearly, which helps researchers understand all kinds of states of ACs. It is also easier to study coordination control strategy with ESS by VESS model.

However, the VESS model is derived directly from the authentic TCL model without simplifying any computation process. The VESS model is built from the point of view of energy storage system to simplify the design and implementation of control strategies. Therefore, the authentic TCL model and the VESS model have the same accuracy.

\subsection{Equivalence of the aggregated TCLs}

The equivalent model of the individual TCL model has been obtained in subsection 2.2. However, the regulation

Table 2 The parameter comparisons between the ESS and the VESS

\begin{tabular}{lll}
\hline Type & ESS model & VESS model \\
\hline Charge and discharge power & $P_{t, c h}^{j}$ and $P_{t, d i s}^{j}$ & $P_{c h}(t)$ and $P_{d i s}(t)$ \\
Capacity & $Q_{t}^{j}$ & $E_{c h}(t)$ and $E_{d i s}(t)$ \\
SOC & $Q_{t}^{j} / Q_{n}^{j}$ & $\frac{E_{c h}}{E_{c h}^{\text {max }}}$ and $\frac{E_{d i s}}{E_{d i s}}$ \\
Efficiency & $\eta_{c h}^{E S S}$ and $\eta_{\text {dis }}^{\text {ESS }}$ & $\eta_{c h}^{V E S S}$ and $\eta_{\text {dis }}^{V E S S}$ \\
\hline
\end{tabular}


power of the individual VESS is small for the power system, which must be aggregated to realize significant role in the power system. There are mainly three equivalent modeling methods of equating aggregated TCLs to the VESS.

A. Direct aggregation method.

The first method is the direct aggregation method, i.e., summarizing the individual TCL directly to calculate the total power of TCLs. Reference [15] establishes the VESS equivalent model by aggregating the power and the stored energy respectively. According to Eq. (12) and (17), the VESS power of the aggregated TCLs can be described as

$$
\begin{aligned}
& P_{T C L s}^{c h}(t)=\sum_{i=1}^{N} P_{c h}^{i}(t) \cdot s_{i}(t) \\
& P_{T C L s}^{d i s}(t)=\sum_{i=1}^{N} P_{d i s}^{i}(t) \cdot s_{i}(t)
\end{aligned}
$$

where $P_{c h}^{i}(t)$ and $P_{d i s}^{i}(t)$ are the charging and discharging power of the $i$-th TCL at time $t$, respectively. $s_{i}(t)$ is equal to 1 when the TCL is participating in demand response, while $s(t)$ is 0 when the TCL stops participating in demand response [42].

According to Eq. (13) and (18), the stored energy of the aggregated TCLs (i.e., the aggregated VESS) can be expressed as

$$
\begin{aligned}
& E_{T C L s}^{c h}(t)=\sum_{i=1}^{N} E_{c h}^{i}(t) \cdot s_{i}(t) \\
& E_{T C L s}^{d i s}(t)=\sum_{i=1}^{N} E_{d i s}^{i}(t) \cdot s_{i}(t)
\end{aligned}
$$

where $E_{c h}^{i}(t)$ and $E_{d i s}^{i}(t)$ are the charging and discharging energy of the $i$-th TCL at time $t$, respectively. Then this model is used to study the features of the VESS by Monte Carlo Simulation. Reference [43] proposes the VESS dynamic model of aggregated TCLs to explore features of the direct load control (DLC) in wind power accommodation. According to Eq. (6) and the total power of $N$ (the number of TCLs) TCLs in Eq. (21) and (22), the aggregation model consists of $N$ indoor temperature changing equations. Because each TCL physical parameters and operating state are different, the Monte Carlo Simulation can be used to show the characteristics of the VESS. It is proved that the VESS can serve as a buffer between supply and demand of the wind power, similar with the traditional ESS. Reference [44] classifies TCLs according to architectural environment and types. Each type of aggregated TCLs is equated to a kind of VESS to make the control easier.

B. Temperature priority list algorithm.
The second method uses temperature priority list (TPL) algorithm [45] to realize the VESS equivalence of the aggregated TCLs. This method divides all TCLs participating in demand response into two groups, the "open" group and the "close" group based on on-off state of the TCLs [46]. In the "open" group, TCLs are ranked from low to high according to their indoor temperature. In the "close" group, TCLs are ranked from high to low. When more TCLs are switched on, the number of the "open" group increases, which can be regarded as charging to the VESS. Otherwise, the VESS is discharging.

Reference $[45,47]$ equates the aggregated TCLs to the VESS by the TPL algorithm to smoothing the microgrid tie-line power fluctuations. Reference [47] defines virtual state of charge (VSOC) to describe the SOC of the VESS based on TPL. Reference [45] defines a state index as $S O C$ to describe the VESS regulation capability based on TPL.

C. State space method.

Based on the Eq. (6), the state space model can also be used to make up the VESS equivalent model of the aggregated TCLs. The complex dynamic process of TCLs is abstracted as a state space expression, as follows.

$$
x(t)=-a x(t)-u(t)
$$

where $x(t) \in \mathbb{R}, u(t) \in \mathbb{R}$, and $a>0$ is a constant. $u(t)$ is the charging or discharging power. $x(t)$ is the SOC of the VESS [48]. However, the parameter $a$ is a big challenge for this method. Reference [49] presents an iterative method based on the Eq. (21) and (22) to identify the VESS model parameter $a$ of TLCs in commercial buildings. Reference $[50,51]$ combines the state space expression and TPL algorithm to obtain the value of $a$.

The above three methods can achieve VESS aggregation. After loads finish participating in demand response and start running again, their operating power may exceed the original power before these loads are controlled, which is called the load rebound phenomenon [8]. During the charging process and the discharging process, the fast change of temperature settings will lead to load rebound of aggregated TCLs. Faced with this issue, some control methods are proposed to reduce load rebound [8].

\section{Control strategies of the VESS in different applications}

Based on the above analysis in section 2, the VESS can play a significant role in the microgrid and the main grid by changing its charging or discharging power. For example, the VESS can help smooth the tie-line power fluctuations, reduce operating costs and increase revenue of the microgrid. Moreover, the VESS is beneficial to regulate frequency, reduce uncertainty from the renewable 
energy and maintain voltage stability in the main network. However, in order to achieve the above beneficial effects, appropriate control strategies are essential. Several main control strategies are shown in Table 3.

\subsection{Control strategies in the microgrid}

Microgrids are composed of distributed micro-sources, power conversion equipment, loads and protection devices. Therefore, microgrids can achieve self-management and self-control [57]. The VESS of TCLs can consuming electricity (i.e. charging) as loads or produce electricity (i.e. discharging) as distributed micro-sources, when their set temperatures are adjusted. Therefore, as a flexible resource, the VESS can balance the deviation between electricity production and consumption to smooth the tie-line power and increase more economic dispatch.

A. Smoothing the tie-line power fluctuations.

Because the electricity generation uncertainties of the renewable energies and electricity consumption uncertainties of loads, there is always deviation of the tie-line power. Reference [52] proposes a coordination control strategy to smooth the tie-line power fluctuations. By setting Butterworth filters with different time constants to smooth the power fluctuations at high and low frequencies [45], the VESS and the ESS can achieve coordinated control. The control strategy guarantees both users' comfort and batteries' lifetime. This control strategy is a traditional filtering algorithm [58], the result of which is not optimal because of the disadvantage of setting time constants only by experience. Continuous state constraints-based optimal control strategy is presented in reference [47], whose objective function is

$$
J=\min \int\left(y_{r e f}-y\right)^{2} d t
$$

where $y_{\text {ref }}$ is expectation deviation between power generation and power consumption and $y$ is the actual value. The method realizes the optimal cooperative control between batteries and the VESS to ensure the microgrid tie-line power is tracked accurately.

B. Economic dispatch.

Due to the energy storage characteristics, the VESS can effectively reduce the cost of power dispatching in the microgrid [59]. Reference [53] proposes an optimal dispatching strategy for the combined cooling-heating-power microgrid considering the VESS to reduce the operating cost of microgrid. Based on the thermal characteristics of buildings, this method adds the VESS model to the microgrid's optimal dispatching model. Moreover, the indoor temperature is maintained within acceptable ranges to ensure users' comfort [60] during economic dispatch.

In addition to considering users' comfort, the price information in the microgrid also needs to be considered to increase economic benefits [61]. Reference [22] presents a dynamic economic dispatch model considering the users' comfort and the real-time electricity price in the hybrid energy microgrid (H-Microgrid). The H-Microgrid is a microgrid integrated with schedulable distribution generators, renewable generations and low-carbon buildings. The dynamic economic dispatch method helps cut down operating costs, reduce times of startup and shutdown for the distribution generators and increase the free capacity of the distribution generators [62].

Due to the small quantity of individual TCL, the VESS in the individual microgrid has less capacity, lower power and greater uncertainty than those in the main network. In order to reduce these disadvantages, the VESS requires coordinated control with generators or other traditional energy storage devices such as the wind power, the photovoltaic and the batteries in the microgrid. Therefore, further research about coordinated control methods is needed.

\subsection{Control strategies in the main network}

Due to uncertainties of renewable energies, inaccuracy of load forecasting, and occurrence of power system failures, the frequency and voltage of the grid are difficult to maintain constant and always in fluctuations [63].

Table 3 The main control strategies of VESS in the previous studies

\begin{tabular}{|c|c|c|}
\hline Application area & Main function & Main control strategies \\
\hline \multirow[t]{2}{*}{ Microgrid } & Smoothing the tie-line power fluctuations & $\begin{array}{l}\text { Coordinated control based on Butterworth filters [52]; } \\
\text { Continuous state constraints-based optimal control strategy [47]. }\end{array}$ \\
\hline & Economic dispatch & $\begin{array}{l}\text { Optimal dispatching strategy considering combined cooling-heating- } \\
\text { power microgrid [53]; } \\
\text { Dynamic economic dispatch model [22]. }\end{array}$ \\
\hline \multirow[t]{3}{*}{ Main network } & Frequency regulation & $\begin{array}{l}\text { Coordination control strategy considering both primary and secondary } \\
\text { frequency regulations [54]; } \\
\text { Priority-stack-based control scheme [46]; } \\
\text { Fuzzy control strategy [44]. }\end{array}$ \\
\hline & Reducing uncertainties from renewable energies & New demand response programs considering VESS [55]. \\
\hline & Maintaining voltage stability & Hierarchical dispatch strategy [56]. \\
\hline
\end{tabular}


The fluctuation smoothing of frequency and voltage mainly depends on traditional power units and reactive power compensation devices. However, frequent adjustments of the generators and reactive power compensation devices will lead to decreasing their lifetime [64]. Therefore, the VESS proposed in this paper is an effective alternative to regulate frequency and maintain voltage stability.

A. Frequency regulation.

As the alternative of traditional frequency regulation tools, VESS can participate in frequency regulation by adjusting the charging and discharging power. A coordination control strategy for the VESS, considering both primary and secondary frequency regulations at the same time, is studied in the reference [54]. The distributed model predictive control is proposed to help the VESS participate in the primary frequency regulation, considering the indoor temperature constraint and switching time constraint. A non-cooperative game is applied to the secondary frequency regulation according to area control error (ACE) [65]. In reference [46], a priority-stack-based control scheme constrained by the VESS no-short-cycling constraint is proposed. The noshort-cycling constraint is the start-up and shutdown time constraints and climbing rate constraint of the VESS. The cases of the paper show this method has a great frequency regulation performance.

Many control strategies are proposed to coordinate the VESS with the traditional ESS. Reference [66] proposes a coordination control method to help the power system frequency regulation. This method firstly combines refrigerators and conventional flywheel energy storage systems into a distributed unit and this unit is equivalent to a VESS. Then all the units are coordinated to maintain the stability of the power system frequency. This control strategy can make the charging and discharging cycles of every VESS minimum to prolong the lifetime of the VESS. Reference [44] proposes a fuzzy control strategy to coordinate the VESS and batteries to regulate the frequency. In order to maintain the frequency stability, fuzzy controllers can adaptively adjust the time constant of filters to change TCLs' set temperature and the output power of the batteries.

B. Reducing uncertainties from renewable energies.

Although the electricity generated by the renewable energies can contribute to energy conservation and emission reduction, their uncertainty reduces the reliability of power systems [67]. Studies show the VESS can help reduce uncertainty from the wind power and photovoltaic. Reference [55] proposes new demand response programs to reduce uncertainty from the renewable energy and improve the economy of the power system operation. The operating revenue equation is established as the objective function, which considers the operating income and cost, the uncertainty from the renewable energy and the prices of the renewable energy. Then the optimal operation mode is gotten by Particle Swarm Optimization method [68], which ensures reducing uncertainty threats by using the VESS can be more economical.

C. Maintaining voltage stability.

Distributed PV generation is susceptible to weather, which leads to voltage fluctuation in the low voltage distribution grids [69]. The VESS can help deal with the voltage fluctuation threat brought about by the distributed PV. A hierarchical dispatch strategy for the VESS is proposed to help maintain voltage stability of the low voltage grids with high solar PV penetration in the reference [56]. This control method includes two levels, the lower level and the upper level. At the lower level, all of VESSs are controlled by the aggregators and at the upper level, aggregators will be controlled by the power system according to the coordination control objective. The cases show that this method has lower cost to smooth the voltage fluctuation than the traditional ESS by making use of the buffering capacity of the VESS.

Besides, the VESS can also show excellent performance by DLC when an emergency occurs in the power system. However, because the demand response in emergency situations is strict about the response speed and the response time for the VESS [70], the control strategies should be improved to meet these requirements.

Many VESS control strategies are based on the assumption that the characteristics of all kinds of VESSs are similar [71, 72]. In fact, the VESS consists of many kinds of TCLs, such as ACs, refrigerators and heat pumps etc., among which they have much differences. Besides, in order to achieve better control results, the VESS is often controlled with traditional ESS to create greater flexibility. Therefore, coordination control strategies [73] between different kinds of VESSs and between the VESS and traditional ESS should be further studied.

\section{Research prospects}

\subsection{Regulation potential estimation of the VESS}

In order to make full use of the VESS, the regulation potential has to be estimated accurately. However, lots of factors can impact the regulation estimation, for example, the different users' willingness, weekday or weekend day, the weather, the ambient temperature and so on [74]. Moreover, after assessing the total capacity of users' TCLs, some subjective and objective factors should also be considered, including the users' comfort [75], users' revenue and power system's control capabilities, etc.

However, the existing estimation methods [76, 77] are difficult to meet all the requirements. The potential assessment methods proposed in most previous papers have some limitations. Many methods of price-based 
demand response estimate ACs potential by electricity price elasticity $[78,79]$, while acquiring accurate price elasticity is a big challenge. The potential estimation methods of incentive-based demand response are mainly based on the direct load control rather than users' willingness $[76,80]$. Therefore, the regulation potential estimation algorithm should be further studied in the future to ensure the accuracy of results, rapidity of calculation and good adaptability.

\subsection{Modeling of heat pumps and refrigerators for VESS}

TCLs include ACs, heat pumps, and refrigerators. However, the studies on TCLs' equivalence to VESS nowadays mainly focus on ACs, because ACs occupy a large proportion of the power consumption during the peak load period and have great regulation potential. Besides, most existing papers about AC models are based on ETP model [14-16], and several references focus on water heaters [13]. Few papers focus on modeling methods of other types of TCLs.

In fact, there is growing potential on heat pumps and refrigerators for residential users [81] and industrial and commercial users [82]. They can also play significant roles in peak shaving, valley filling, system frequency regulating, and uncertainty restraining brought by RESs, as what ACs have done [33]. The heat pumps and refrigerators have similar physical properties and dynamic processes with ACs. However, there still exists many differences between heat pumps, refrigerators and ACs. On one hand, the energy stored in hot water provided by heat pumps will be dissipated through pipes, and lost when hot water is used. This special energy transfer process increases complexity of heat balance equation. Besides, heat pumps are widely used in industry and commerce with high power but small quantity [83]. Refrigerators are mainly used by residential users with lower power but larger quantity. These result in differences of heat pumps, refrigerators and ACs in modeling and control strategies [84].

\subsection{Hierarchical coordinated control methods of the VESS}

At present, TCLs participate in demand response mainly by the centralized control method $[85,86]$, while decentralized control method is rarely used [87]. However, due to the limitation of communication capability [85], it is difficult to achieve centralized control of massive VESSs [86]. Faced with this issue, the hierarchical coordinated control method $[88,89]$ can probably solve this problem well.

The hierarchical coordinated control method usually divides the whole control process into two levers. In the lower level, all the VESS units are divided into different groups controlled by different aggregators. In the upper level, all the aggregators will receive control signal sent by the power system operator. This control mode can greatly reduce the demand for communication capacity since the power system only control aggregators rather than every VESS. However, how to choose the certain VESS units for a certain aggregator to reduce the control pressure of the lower lever is a challenge. Moreover, another difficulty is how to control VESS units or aggregators to participate in demand response more economically and more efficiently.

\subsection{Control strategies considering VESS, ESS and RESs}

The VESS modeling method makes it easier for TCLs to participate in DR. As a special ESS, VESS has similar external electrical characteristics with traditional ESS, which can achieve the time shift of power usage [51]. Besides, due to the increasing penetration of RES, it will be a hotspot to combine VESS with ESS from the point of view of ESS, considering the uncertainties of RESs.

However, there are still several challenges to be dealt with. Because the ESS is relatively centralized and has large capacity, fast control speed and high precision can be realized, which helps ESS participate in RES control [90-92]. However, it is geographically dispersed for the VESS. The control method considers not only the electrical limitations, but also the comfort and willingness of users. Due to more constraints and huger quantities, fast control of VESS is more difficult to achieve. Therefore, better control strategies need to be proposed to meet the requirements of the RES control.

\subsection{Reliability and fast response in frequency control for VESS}

As a special ESS, VESS has obvious advantages in the field of frequency control for power systems, considering two reasons: on one hand, the number of VESSs is huge and VESSs can provide huge power reserve, especially in summer and winter. On the other hand, because it can take advantage of a large number of existing TCLs, it is less expensive for VESS to participate in frequency control.

However, a series of problems still need to be solved $[44,46,54,65]$. The VESS is geographically dispersed, and it is controlled through sophisticated communication systems to achieve fast response. Therefore, the most serious problem is the reliability of VESS as a reserve for frequency control since more components in the system will lead to lower reliability [93]. Besides, frequency control requires fast response and the communication delay should also be considered. Not only the progress of control strategies, but also the improvement of hardware needs to be focused on.

\section{Conclusions}

The penetration of RESs is increasing rapidly, while it brings more uncertainties and leads to power and 
voltage fluctuations. As an alternative to traditional generators, TCLs can provide regulation capacities to deal with these challenges by demand response. This paper focuses on summarizing the VESS equivalent modeling methods of the TCLs and the control strategies of the VESS. First, The VESS equivalence of the individual TCL and the aggregated TCLs are studied. The VESS is compared with traditional ESS about physical parameters. Then, the control strategies of the VESS in microgrids and the main network are showed. The VESS reveals excellent performance in frequency regulation, maintaining voltage stability and economic dispatch.

Although there are a lot of research on the VESS, the study of the VESS is still in its infancy, and many problems need to be solved. First, the accuracy of regulation potential estimation needs to be improved to make full use of the VESS. Besides, the VESS equivalent modeling method of the heat pumps and refrigerators should be studied since they have increasing potential to participate in the demand response. Then, hierarchical coordinated control strategies comprehensively considering VESS, ESS and RESs need to be studied further. Finally, reliability and fast response of VESS in frequency control should be focused on.

\begin{abstract}
Abbreviations
ACE: Area control error; ACs: Air conditionings; COP: Coefficient of performance; DLC: Direct load control; DR: Demand response; EER: Energy efficiency ratio; ESS: Energy Storage system; ETP: Equivalent thermal parameter; H-Microgrid: Hybrid energy microgrid; PV: Photovoltaic; RESs: Renewable energy sources; SOC: State of charge; TCL: Thermostatically controlled load; TPL: Temperature priority list; VESS: Virtual energy storage system; VSOC: Virtual state of charge
\end{abstract}

\section{Acknowledgements}

Not applicable.

\section{Authors' contributions}

Kang Xie, as the first author, carried out the literature review and drafted the manuscript. Hongxun Hui, as the second author, contributed significantly to manuscript preparation and manuscript revision. Yi Ding, as the corresponding author, conceived of the manuscript and revised the draft of the manuscript. All authors read and approved the final manuscript.

\section{Authors' information}

K. Xie (1995-), male, Ph.D. Candidate of Zhejiang University in China, Major in the demand side management.

H. Hui (1992-), male, Ph.D. Candidate of Zhejiang University in China, Major in the demand side management.

Y. Ding (1978-), male, Ph.D. and Professor of Zhejiang University in China, Major in the power system reliability, the electricity market and the smart grid.

\section{Funding}

This work is supported in part by the National Key Research and Development Program of China under Grant 2016YFB0901100, and in part by the National Natural Science Foundation of China (NSFC) under Grant 51577167.

\section{Availability of data and materials}

Data sharing is not applicable to this article as no datasets were generated or analyzed during the current study.

\section{Competing interests}

The authors declare that they have no competing interests.
Received: 22 August 2019 Accepted: 9 October 2019

Published online: 04 December 2019

\section{References}

1. Zhang, X. H., Zhao, J. Q., \& Chen, X. Y. (2011). Multi-objective unit commitment modeling and optimization for energy-saving and emission reduction in wind power integrated system. Power System Protection and Control, 39(17), 33-39.

2. Wang, M., Mu, Y., Jia, H., Wu, J., Yu, X., \& Qi, Y. (2017). Active power regulation for large-scale wind farms through an efficient power plant model of electric vehicles. Applied Energy, 185, 1673-1683.

3. Liao, Z., Chen, S., \& Lin, C. (2018). Distribution network voltage state assessment with distributed generation based on improved probabilistic power flow method. In DEStech Transactions on Environment, Energy and Earth Sciences, (appeec).

4. China Reform Daily. The accumulative installed scale of hydropower, wind power and photovoltaic power in China ranks first in the world in 2018 [EB/ OL]. http://www.cspplaza.com/article-15572-1.html, 2019-07-04.

5. Sina Southern Energy. Construction of renewable energy power generation exceeding 1/4 peak shaving power supply in 2018 is accelerated [EB/OL] http://gd.sina.cn/energy/2019-06-28/detail-ihytcitk8214889.d.html?vt= $4 \& w m=2256 \_3664 \& c i d=183609,2019-06-28$.

6. Ren, S., Yang, X., Zhang, Y., Zhao, B., Xie, L., \& Weng, G. (2017). A real time optimization strategy for microgrid integrated with schedulable ability and uncertainties. In In Proceedings of the CSEE.

7. Huang, W., Zhang, N., Kang, C., Li, M., \& Huo, M. (2019). From demand response to integrated demand response: Review and prospect of research and application. Protection and Control of Modern Power Systems, 4(1), 12.

8. Cui, W., Ding, Y., Hui, H., Lin, Z., Du, P., Song, Y., \& Shao, C. (2018). Evaluation and sequential dispatch of operating reserve provided by air conditioners considering Lead-lag rebound effect. IEEE Transactions on Power Systems, 33(6), 6935-6950.

9. Wang, Y., Chen, Q., Kang, C., Zhang, M., Wang, K., \& Zhao, Y. (2015). Load profiling and its application to demand response: A review. Tsinghua Science and Technology, 20(2), 117-129.

10. Siano, P. (2014). Demand response and smart grids-A survey. Renewable and Sustainable Energy Reviews, 30, 461-478.

11. Bie, Z., Xie, H., Hu, G., \& Li, G. (2015). Optimal scheduling of power systems considering demand response. Journal of Modern Power Systems and Clean Energy, 4(2), 180

12. Xie, K., Zhang, K., Luan, K., et al. (2019). Exploration of demand response score scheme under the background of electric power system reform. Power Demand Side Management, 21(03), 7-10.

13. Qi, Y., Wang, D., Jia, H., Chen, N., Wei, W., Liu, K., \& Fan, M. (2017). Research on under frequency load shedding strategy using aggregated thermostatically controlled loads based on demand response. Proceedings of the CSEE, 751-760.

14. Li, N., \& Wang, X. (2015). Research of air conditioners providing frequency controlled reserve for microgrid. Power Syst Prot Control, 43, 101-105.

15. Wang, Y., Tong, Y., \& Huang, M. (2017). Research on virtual energy storage model of air conditioning loads based on demand response. Power System Technology, 41(2), 394-401.

16. Hui, H., Ding, Y., Lin, Z. Z., Siano, P., \& Song, Y. (2019). Capacity allocation and optimal control of inverter air conditioners considering area control error in multi-area power systems. IEEE Transactions on Power Systems.

17. Song, M., Gao, C., \& Su, W. (2016). Modeling and controlling of airconditioning load for demand response applications. Autom Electr Power Syst, 40(14), 158-167.

18. Ding, Y., Song, Y. H., Hui, H., \& Shao, C. (2019). Integration of air conditioning and heating into modern power systems: enabling. Springer.

19. Qingze, W., Xingying, C., Qingguo, Y., Shiming, X., \& Yingchen, L. (2017). Two-layer flexible optimal strategy for air-conditioning of office building under TOU price [J]. Power System Protection and Control, 45(21), 43-50.

20. Hui, H., Ding, Y., \& Zheng, M. (2018). Equivalent modeling of inverter air conditioners for providing frequency regulation service. IEEE Transactions on Industrial Electronics, 66(2), 1413-1423.

21. Xiao, H. , Ming, Z. , \& Gengyin, L. I. . (2018). Multi-objective optimal dispatching of active distribution networks considering energy storage systems and air-conditioning loads. Power System Protection and Control.

22. Jin, X., Mu, Y., Jia, H., Wu, J., Jiang, T., \& Yu, X. (2017). Dynamic economic dispatch of a hybrid energy microgrid considering building based virtual energy storage system. Applied Energy, 194, 386-398. 
23. Soroudi, A., Siano, P., \& Keane, A. (2015). Optimal DR and ESS scheduling for distribution losses payments minimization under electricity price uncertainty. IEEE Transactions on Smart Grid, 7(1), 261-272.

24. Jianlin, L. I., Huimeng, M. A., Xiaodong, Y., Zhan, W., \& Le, G. E. (2017). Overview on key applied technologies of large-scale distributed energy storage. Power System Technology.

25. Wei, L., Zhao, B., Wu, H., \& Zhang, X. (2015). Optimal allocation model of energy storage system in virtual power plant environment with a high penetration of distributed photovoltaic generation. Autom. Electr Power Syst, 23, 66-74.

26. Lei, F., Yugang, N. , Siming, W. , \& Tinggang, J. . (2018). Optimal capacity determination method based on day-ahead scheduling and real-time control. Power System Protection and Control.

27. Byrne, R. H., Nguyen, T. A., Copp, D. A., Chalamala, B. R., \& Gyuk, I. (2017). Energy management and optimization methods for grid energy storage systems. IEEE Access, 6, 13231-13260.

28. Meng, L. I. U., LIANG, W., \& ZHANG, Y. (2017). Cooperative generation-load optimal dispatching model considering airconditioning load group control. Power System Technology, 41(4), 1230-1236.

29. Wang, D., Zeng, R., \& Mu, Y. (2015). An optimization method for new energy utilization using thermostatically controlled appliances. Power System Technology, 39(12), 3457-3462.

30. Chenxing, Y. A. N. G., Qingshan, X. U., \& Xufang, W. A. N. G. (2017). Strategy of constructing virtual peaking unit by public buildings' central air conditioning loads for day-ahead power dispatching. Journal of Modern Power Systems and Clean Energy, 5(2), 187-201.

31. Hui, H., Ding, Y., Liu, W., Lin, Y., \& Song, Y. (2017). Operating reserve evaluation of aggregated air conditioners. Applied Energy, 196, 218-228.

32. Xie, D., Hui, H., Ding, Y., \& Lin, Z. (2018). Operating reserve capacity evaluation of aggregated heterogeneous TCLs with price signals. Applied Energy, 216, 338-347.

33. lacovella, S., Ruelens, F., Vingerhoets, P., Claessens, B., \& Deconinck, G. (2015). Cluster control of heterogeneous thermostatically controlled loads using tracer devices. IEEE Transactions on Smart Grid, 8(2), 528-536.

34. Zhao, H., Wu, Q., Huang, S., Zhang, H., Liu, Y., \& Xue, Y. (2016). Hierarchical control of thermostatically controlled loads for primary frequency support. IEEE Transactions on Smart Grid, 9(4), 2986-2998.

35. Ning, L. (2012). An evaluation of the hvac load potential for providing load balancing service. IEEE Transactions on Smart Grid, 3(3), 1263-1270.

36. Ding, Y., Cui, W., Zhang, S., Hui, H., Qiu, Y., \& Song, Y. (2019). Multi-state operating reserve model of aggregate thermostatically-controlled-loads for power system short-term reliability evaluation. Applied Energy, 241.

37. Hui, H., Ding, Y., \& Yang, S. (2019). Modeling and analysis of inverter air conditioners for primary frequency control considering signal delays and detection errors. Energy Procedia, 158, 4003-4010.

38. Kim, Y. J., Norford, L. K., \& Kirtley, J. L. (2014). Modeling and analysis of a variable speed heat pump for frequency regulation through direct load control. IEEE Transactions on Power Systems, 30(1), 397-408.

39. Kim, Y. J., Fuentes, E., \& Norford, L. K. (2015). Experimental study of grid frequency regulation ancillary service of a variable speed heat pump. IEEE Transactions on Power Systems, 31(4), 3090-3099.

40. Song, M., Gao, C., Yang, J., \& Yan, H. (2018). Energy storage modeling of inverter air conditioning for output optimizing of wind generation in the electricity market. CSEE Journal of Power and Energy Systems, 4(3), 305-315.

41. Jin, X., Wu, J., Mu, Y., Wang, M., Xu, X., \& Jia, H. (2017). Hierarchical microgrid energy management in an office building. Applied Energy, 208, 480-494.

42. Khan, S., Shahzad, M., Habib, U., Gawlik, W., \& Palensky, P. (2016). Stochastic battery model for aggregation of thermostatically controlled loads. In 2016 IEEE International Conference on Industrial Technology (ICIT) (pp. 570-575). IEEE.

43. Ai, X., Zhao, Y., \& Zhou, S. (2016). Study on virtual energy storage features of air conditioning load direct load control. Proceedings of the CSEE, 36(6), 1596-1603.

44. Congwei, T. U. , Jun, C. , Dongli, Y. U. , \& Xiangyang, M. . (2019). Control strategy of virtual energy storage system participating in frequency modulation based on air conditioning loads. Power Demand Side Management.

45. Wang, D., Ge, S., Jia, H., Wang, C., Zhou, Y., Lu, N., \& Kong, X. (2014). A demand response and battery storage coordination algorithm for providing microgrid tie-line smoothing services. IEEE Transactions on Sustainable Energy, 5(2), 476-486.
46. Sanandaji, B. M., Hao, H., Poolla, K., \& Vincent, T. L. (2014, June). Improved battery models of an aggregation of thermostatically controlled loads for frequency regulation. In 2014 American Control Conference (pp. 38-45). IEEE.

47. Zhaoyu, C. H. E. N., Dan, W. A. N. G., \& Hongjie, J. (2017). Optimal smoothing control strategy of virtual energy storage system in microgrid based on continuous state constraints. Power System Technology, 41(1), 55-63.

48. Hao, H., Sanandaji, B. M., Poolla, K., \& Vincent, T. L. (2014). Aggregate flexibility of thermostatically controlled loads. IEEE Transactions on Power Systems, 30(1), 189-198.

49. Hughes, J. T., Domínguez-García, A. D., \& Poolla, K. (2015). Virtual battery models for load flexibility from commercial buildings. In 2015 48th Hawaii International Conference on System Sciences (pp. 2627-2635). IEEE.

50. Zhao, L., Zhang, W. (2016). A geometric approach to virtual battery modeling of thermostatically controlled loads. In 2016 American Control Conference (ACC) (pp. 1452-1457). IEEE.

51. Hao, H., Sanandaji, B. M., Poolla, K., \& Vincent, T. L. (2013). A generalized battery model of a collection of thermostatically controlled loads for providing ancillary service. In 2013 51st Annual Allerton Conference on Communication, Control, and Computing (Allerton) (pp. 551-558). IEEE.

52. Ran, W., Dan, W., Hongjie, J., Zhanyong, Y., \& Yebai, Q. I. (2015). A coordination control strategy of battery and virtual energy storage to smooth the micro-grid tie-line power fluctuations. Proceedings of the CSEE, 35(20), 5124-5134.

53. Jin, X., Mu, Y., Jia, H., Yu, X., \& Chen, N. (2017). Optimal scheduling method for a combined cooling, heating and power building microgrid considering virtual storage system at demand side. In Proceedings of the CSEE (Vol. 37, no. 2, pp. 581-590).

54. Zheng, Y., Hill, D., Liu, T., \& Meng, K. (2018). Supplementary frequency regulation with multiple virtual energy storage system aggregators. Electric Power Components and Systems, 46(16-17), 1719-1730.

55. Hakimi, S. M., \& Tafreshi, S. M. (2016). Smart virtual energy storage control strategy to cope with uncertainties and increase renewable energy penetration. Journal of Energy Storage, 6, 80-94.

56. Wang, D., Meng, K., Gao, X., Qiu, J., Lai, L. L., \& Dong, Z. Y. (2017). Coordinated dispatch of virtual energy storage systems in LV grids for voltage regulation. IEEE Transactions on Industrial Informatics, 14(6), 2452-2462

57. Yongiian, Y., Weixin, L., Wenging, Y., Baosheng, W., Guangshan, L., Gangwei, Y., \& Le, K. (2018). Research on dynamic adaptive droop control strategy for microgrid. In 2018 2nd IEEE Conference on Energy Internet and Energy System Integration (EI2) (pp. 1-4). IEEE.

58. Zhang, Y., Guo, L., Jia, H., Li, Z., \& Lu, Z. (2012). An energy storage control method based on state of charge and variable filter time constant [J]. Automation of Electric Power Systems, 6.

59. Zhang, Z., Guo, X., Zhang, X., \& Wu, D. (2017). Strategy of smoothing wind power fluctuation based on storage battery. Power System Protection and Control, 3, 62-68.

60. Tong, Y., You, X., Wang, Y., \& Huang, M. (2017). Research on virtual energy storage of air conditioning load. Journal of Beijing Jiaotong University, 41(5), 126-131.

61. Chassin, D. P., Stoustrup, J., Agathoklis, P., \& Dijilali, N. (2015). A new thermostat for real-time price demand response: Cost, comfort and energy impacts of discrete-time control without deadband. Appl Energy, 155, 816-825.

62. Zhao, Y., Lu, Y., Yan, C., \& Wang, S. (2015). MPC-based optimal scheduling of grid-connected low energy buildings with thermal energy storages. Energy and Buildings, 86, 415-426.

63. Shi, Q., Cui, H., Li, F., Liu, Y., Ju, W., \& Sun, Y. (2017). A hybrid dynamic demand control strategy for power system frequency regulation. CSEE Journal of Power and Energy Systems, 3(2), 176-185.

64. Shi, Q., Li, F., Hu, Q., \& Wang, Z. (2018). Dynamic demand control for system frequency regulation: Concept review, algorithm comparison, and future vision. Electr Power Syst Res, 154, 75-87.

65. Dong, D., Zongqi, L., \& Shuili, Y. (2015). Battery energy storage aid automatic generation control for load frequency control based on fuzzy control. Power System Protection and Control, 43(8), 81-87.

66. Cheng, M., Sami, S. S., \& Wu, J. (2017). Benefits of using virtual energy storage system for power system frequency response. Appl Energy, 194, 376-385.

67. Shu, Y., Zhang, Z., Guo, J., \& Zhang, Z. L. (2017). Study on key factors and solution of renewable energy accommodation. Proceedings of the CSEE, $37(1), 1-8$. 
68. Yuhang, X., Junyong, L., Chao, F., Xiaoyu, L., Chen, W. U., \& Zhengwen, H. (2016). Optimal scheduling model of virtual power plant considering demand response. Power System Technology.

69. Li, S., Jiang, C., Zhao, Z., \& Li, Z. (2017). Study of transient voltage stability for distributed photovoltaic power plant integration into low voltage distribution network. Power System Protection and Control, 45(8), 67-72.

70. Bijun, L. I., \& Yuqiang, H. (2016). Research of emergency load regulation for security and stability control [J]. Power System Protection and Control, 44(11), 104-110.

71. Mehta, N., Sinitsyn, N. A., Backhaus, S., \& Lesieutre, B. C. (2014). Safe control of thermostatically controlled loads with installed timers for demand side management. Energy Conversion and Management, 86, 784-791.

72. Liu, M., Shi, Y., \& Liu, X. (2015). Distributed MPC of aggregated heterogeneous thermostatically controlled loads in smart grid. IEEE Transactions on Industrial Electronics, 63(2), 1120-1129.

73. Vrettos, E., Tang, Y., Xu, Y., \& Xu, Y. (2019). A distributed control scheme of thermostatically controlled loads for the building-microgrid community. IEEE Transactions on Sustainable Energy.

74. Li, W., Lian, J., Engel, D., \& Wang, H. (2018). Ensemble-based uncertainty quantification for coordination and control of thermostatically controlled loads. Journal of Control and Decision, 5(2), 148-168.

75. Yang, J., Liu, T., Wang, H., Tian, Z., \& Liu, S. (2019). Optimizing the regulation of aggregated thermostatically controlled loads by jointly considering consumer comfort and tracking error. Energies, 12(9), 1757.

76. Wang, B., Zhu, F., Ji, W., \& Cao, Y. (2016). Load cutting potential modeling of central air-conditioning and analysis on influencing factors. Automation of Electric Power Systems, 40(19), 44-52.

77. Yin, R., Kara, E. C., Li, Y., DeForest, N., Wang, K., Yong, T., \& Stadler, M. (2016). Quantifying flexibility of commercial and residential loads for demand response using setpoint changes. App/ Energy, 177, 149-164.

78. Yaping, L. I. , Ke, W. , Xiaorui, G. , Dan, Z. , \& Wenbo, M. . (2015). Demand response potential based on multi-scenarios assessment in regional power system. Power System and Clean Energy.

79. Wang Yuan; Zhou Ming; State Key Laboratory of Alternate Electrical Power System with Renewable Energy Sources ( North China Electric Power University ); (2017). Demand response potential evaluation method of timeof-use price for residential community. Electric Power Construction.

80. Yuchao, Z., Jianxue, W., \& Xiaoyu, C. (2018). Direct control strategy of central air-conditioning load and its schedulable potential evaluation. Electric Power Automation Equipment, 38(5), 227-234.

81. Alimohammadisagvand, B., Jokisalo, J., Kilpeläinen, S., Ali, M., \& Sirén, K. (2016). Cost-optimal thermal energy storage system for a residential building with heat pump heating and demand response control. Appl Energy, 174, 275-287.

82. Schibuola, L., Scarpa, M., \& Tambani, C. (2015). Demand response management by means of heat pumps controlled via real time pricing. Energy and Buildings, 90, 15-28.

83. Wang, D., Fan, M., \& Jia, H. (2014). User comfort constraint demand response for residential thermostatically-controlled loads and efficient power plant modeling. Proceedings of the CSEE, 34(13), 2071-2077.

84. Wang, J. X., Zhong, H. W., Xia, Q., \& Yang, S. (2016). Model and method of demand response for thermostatically-controlled loads based on costbenefit analysis. Automation of Electric Power Systems, 40(5), 45-53.

85. Vanouni, M., \& Lu, N. (2014). Improving the centralized control of thermostatically controlled appliances by obtaining the right information. IEEE Transactions on Smart Grid, 6(2), 946-948.

86. Lu, N., \& Zhang, Y. (2012). Design considerations of a centralized load controller using thermostatically controlled appliances for continuous regulation reserves. IEEE Transactions on Smart Grid, 4(2), 914-921.

87. Molina-Garcia, A., Bouffard, F., \& Kirschen, D. S. (2010). Decentralized demand-side contribution to primary frequency control. IEEE Trans Power Syst, 26(1), 411-419.

88. Tindemans, S. H., Trovato, V., \& Strbac, G. (2015). Decentralized control of thermostatic loads for flexible demand response. IEEE Transactions on Control Systems Technology, 23(5), 1685-1700.

89. Vrettos, E., Ziras, C., \& Andersson, G. (2016). Fast and reliable primary frequency reserves from refrigerators with decentralized stochastic control. IEEE Transactions on Power Systems, 32(4), 2924-2941.

90. Shilei, D., Mingyu, W. . (2018). Cooperative control strategy of dc microgrid power flow controller and distributed energy storage system. Power System Protection and Control.
91. Jinzhou, F. U., Ming, S. . (2018). Energy management strategy based on weather condition for photovoltaic-energy storage integrated power system. Power System Protection and Control.

92. Zilong, Y., Zhenhao, S., Jing, P., et al. (2015). Multi-mode coordinated control strategy of distributed PV and energy storage system. Proceeding of the CSEE, 39(8), 2213-2220

93. Jia, H., Ding, Y., Song, Y., Singh, C., \& Li, M. (2018). Operating reliability evaluation of power systems considering flexible reserve provider in demand side. IEEE Transactions on Smart Grid, 10(3), 3452-3464.

\section{Submit your manuscript to a SpringerOpen ${ }^{\circ}$ journal and benefit from:}

- Convenient online submission

- Rigorous peer review

- Open access: articles freely available online

- High visibility within the field

- Retaining the copyright to your article

Submit your next manuscript at $>$ springeropen.com 